\title{
Evolution of volatiles from Europa's interior into its ocean
}

\author{
M. MELWANI DASWANI ${ }^{1 *}$, S. D. VANCE ${ }^{1}$
}

${ }^{1}$ Jet Propulsion Laboratory, California Institute of

Technology, Pasadena, CA 91101, USA

(*correspondence: daswani@jpl.caltech.edu)

\section{Introduction and Methods}

Jupiter's moon Europa hosts a $>100 \mathrm{~km}$ deep ocean beneath its $3-30 \mathrm{~km}$ ice shell [e.g. 1]. Key to understanding the habitability of Europa's ocean is its composition and origin. We explore an endogenous origin for Europa's earliest ocean. Specifically, we consider ocean build-up by volatile exsolution from volatile-bearing mineral destabilization, caused by thermal metamorphism during planetary differentiation and Europa's tidal-orbital evolution in the Jovian system. We use Gibbs energy minimization program Perple_X [2] to model the stability of minerals and fluids, and quantify the mass and composition of exsolved volatiles.

\section{Results and Discussion}

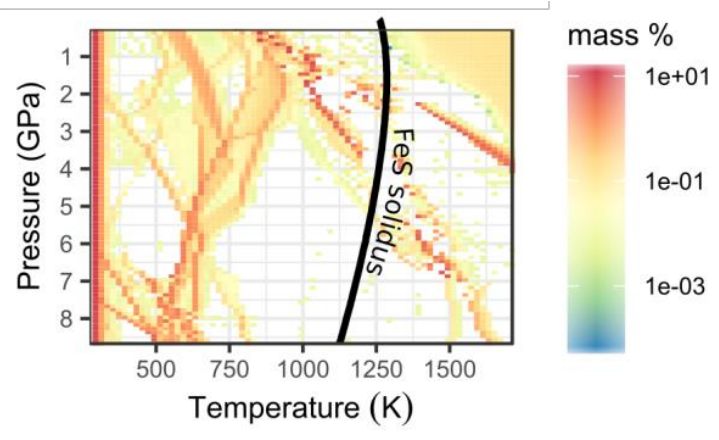

Figure 1: Mass percent of fluid and melt extracted from the rocky interior of Europa as a result of isobaric heating in the interior. The minimum temperature achieved in Europa's interior is the FeS solidus, because Europa is differentiated; thus, we constrain a minimum mass of exsolved fluid.

Testing different initial chondritic compositions and retained-to-extracted fluid ratios, we find that Europa's ocean could be entirely built from volatile (mainly $\mathrm{H}_{2} \mathrm{O}$ ) exsolution, and may have evolved from sulfate- to chloride-rich from accretion until the present day. [JPL-Caltech under contract from NASA. Government sponsorship acknowledged.]

[1] Hendrix, A. R. et al. (2018). The NASA Roadmap to Ocean Worlds. Astrobiology, 19(1), 1-27. [2] Connolly J. A. D. (2009) $G^{3}$ 10, Q10014. 